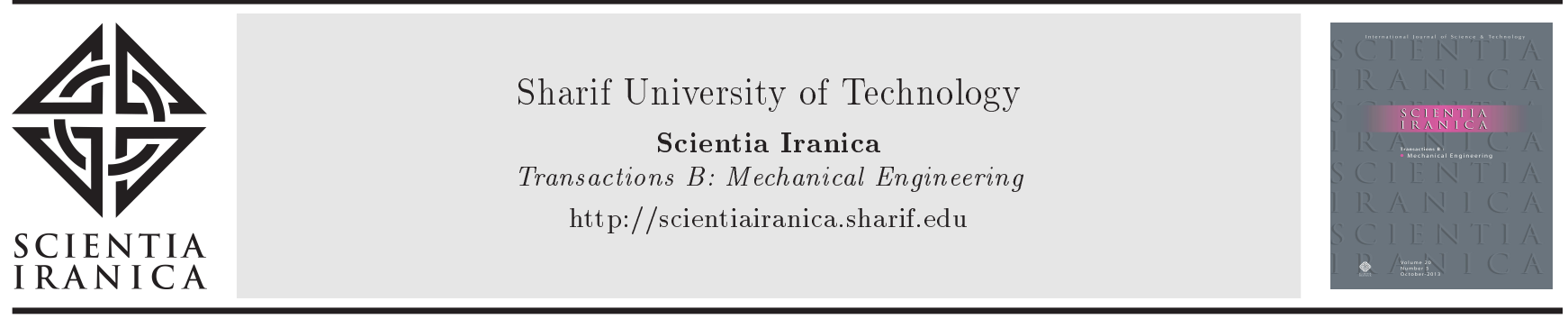

\title{
Pulsating flow induced parametric instabilities of a smart embedded micro-shell based on nonlocal piezoelasticity theory
}

\author{
V. Atabakhshian and A. Shooshtari* \\ Department of Mechanical Engineering, Faculty of Engineering, Bu-Ali Sina University, Hamedan, Iran.
}

Received 21 January 2018; received in revised form 16 September 2018; accepted 29 December 2018

\section{KEYWORDS \\ Dynamical instability; \\ Pulsating flow; \\ Smart materials; \\ Cylindrical shell; \\ Nonlocal theory.}

\begin{abstract}
In this study, the dynamical instabilities of a smart embedded micro-shell conveying pulsating fluid flow are investigated based on nonlocal piezoelasticity theory and nonlinear cylindrical shell model. The micro-shell is surrounded by an elastic foundation, which is suitable for both Winkler spring and Pasternak shear modules. The internal fluid flow is considered to be purely harmonic, irrotational, isentropic, Newtonian, and incompressible and is mathematically modeled using linear potential flow theory, time mean Navier Stokes equations, and Knudsen number. To bring the micro-scale problem closer to the reality, the pulsating viscous effects and the slip boundary condition are also taken into account. Employing the modified Lagrange equations of motion for open systems, the nonlinear coupled governing equations are achieved and, consequently, the instability boundaries are obtained using Bolotin's method. In the section of numerical results, a comprehensive discussion about the dynamical instabilities of the system is presented (such as divergence, flutter, and parametric resonance). It was found that the application of the positive electric potential field would improve the stability of the system as an actuator or as a vibration amplitude controller in the micro electromechanical systems.
\end{abstract}

(C) 2020 Sharif University of Technology. All rights reserved.

\section{Introduction}

Undoubtedly, nanotechnology is and will be the basis of the future industrial revolution, and the discovery of nanotubes is one of the major improvements that can speed up this revolution. Generally, nanotechnology involves the construction and application of biological, chemical, and physical systems, ranging from micro to nanometer. Nowadays, fluid-conveying nanotubes are actively researched and studied due to their extensive possible applications in biological systems (e.g., biological separation, biosensing, molecular imaging),

*. Corresponding author. Tel.: +98 8138272410 E-mail address: shooshta@basu.ac.ir (A. Shooshtari)

doi: $10.24200 /$ sci.2018.50293.1627 medicine (e.g., drug delivery), chemistry (e.g., fuel storing cells, chemical analyses), physics (e.g., optical structures), and other research areas [1-3].

The dynamic and instability analyses of fluidconveying pipes are one of the important challenges in the field of fluid conveying systems, which basically started by the Trans-Arabian pipeline project in 1950 [4]. Certainly, the widespread applications of pipes conveying or containing fluids in various industries such as oil and gas, water and sewage, military and aerospace, and medicine and chemistry are enough to attract researchers' attention in this regard. Some of the valuable pioneering works are as follows.

Paidoussis [5] examined fluid and body interactions in slender structures that convey axial flows. He modeled and analyzed the dynamics and instabilities of fluid-conveying systems. Amabili [6] studied nonlinear 
dynamics and instabilities of circular cylindrical shells conveying fluid and classified fluid instabilities for them. He utilized various nonlinear shell models, modal expansion analyses, and energy approaches to present a comprehensive analysis of the problem. Dynamical behavior of the fluid-conveying pipes based on various types of beam theories was investigated by Reddy and Wang [7]. They obtained equation of motion via energy approaches, in which the finite element method was employed to solve the problem. Pellicano and Amabili [8] studied dynamics and instabilities of empty and fluid-filled circular cylindrical shells under external loadings. They used modal analysis approach to simulate the problem. Sadeghi and Karimi-Dona [9] employed Finite Element Method (FEM) and the state space approach besides MATLAB program to model the dynamical behaviors of fluid-conveying pipes with a moving sprung mass on it. Gu et al. [10] used the integral transform technique to obtain the dynamical response of fluid-conveying pipes.

In the micro- and nano-scale problems and in the field of biomechanics, the flutter phenomenon of veins conveying blood flow was investigated by Kamm and Pedley [11]. Paidoussis [12] proposed a model for the Fluid-Structure Interaction (FSI) between the blood flow and veins. Moreover, in the field of nano mechanics, Yan et al. [13] studied the stability and critical flow velocity of multi-walled carbon nanotubes (CNTs) conveying fluid. Nonlinear vibration analysis of double-walled CNTs conveying fluid was carried out by Kuang et al. [14]. Ghorbanpour Arani et al. [15] studied the flow-induced vibration and instability of a smart polymeric micro tube subjected to the axial electric field. They found that the imposition of electric potential field had a significant effect on the stability of the smart micro tube. In another work, Ghorbanpour Arani et al. [16] studied the nonlinear dynamical response of embedded fluid-conveyed micro-tubes reinforced by Boron nitride nanotubes (BNNTs). In other works, Ghorbanpour Arani et al. $[17,18]$ studied the dynamic stability of double-walled BNNTs conveying viscose fluid. Atabakhshian et al. [19] studied nonlinear dynamic and instability of an elastically coupled CNTPPB system with internal flow. They proposed an elastically coupled nano-beam system composed of CNT conveying fluid and Piezoelectric Polymeric Beam (PPB) polarized in the axial direction. They showed that this system would improve the stability of CNT conveying fluid by applying positive electric potential to the coupled PPB.

In all of the above investigations, a constant flow velocity has been considered inside a structure, which is not suitable for many empirical problems like those with pumps or turbines in the pipelines. Amongst the time-dependent flow regimes inside a circular cylindrical shell, pulsating flow with purely harmonic nature has attracted much attention amongst researchers due to its important applications in industries. Practically, special pumps generate pulsating flow (e.g., beating pumps in biomechanics) and, arbitrarily, a wide range of applications are classified as purely harmonic irrespective of small velocity perturbations. Moreover, a general time-dependent flow regime may be decoupled into harmonic components as a Fourier series and, in such cases, the analysis may be considered for a single Fourier component [20]. In this regard, nonlinear dynamics of pipes conveying pulsating fluid with a combination of parametric and internal resonances was studied by Panda and Kar [21]. Azrar et al. [22] studied the dynamic and parametric instabilities of singlewalled CNTs conveying pulsating and viscous fluid. Liang and $\mathrm{Su}$ [23] utilized the averaging method for the stability analysis of single-walled carbon nanotubes conveying pulsating and viscous fluid. Pipes conveying pulsating fluid and exposed to the external vortex were analyzed by Da et al. [24]. They employed multiple time-scale method to obtain the parametric resonances of the system. Nonlinear dynamics of simply supported pipes with motion constraints and internal pulsating fluid was investigated by Wang [25]. Recently, micro pulsating fluid flow in heat pipes with alternate withes was examined by Yang et al. [26]. Tubaldi et al. [27] modeled nonlinear dynamics of shells subjected to pulsating fluid flow. They utilized a special pulsatile time-dependent function for blood flow velocity based on the heart beating period. They also recently examined in another work [28] the effects of pulse-wave propagation on nonlinear dynamics of a shell conveying pulsatile flow.

The simulation of the small-scale structures is usually done using two main categories: the Molecular Dynamics (MD) method and the classical continuum mechanics. It is clear that the MD method is a costly and time-consuming method whose application is limited to smaller nanostructures with a few molecules. Contrary to the MD method, the nonlocal elasticity theory was later developed [29] for larger scale nano/micro-structures based on classical continuum mechanics such as classical Euler Bernoulli beam, Timoshenko beam, and shell theories [30-34].

Based on the above survey, the lack of proper research on instability prediction and instability smart control of fluid-conveying microtubes can be clearly felt. Hence, this study investigates the flow-induced vibrations and instabilities of a smart embedded microshell conveying pulsating fluid, as schematically shown in Figure 1. Parametric excitation due to harmonic pulsation in the flow velocity, Knudsen number effects, pulsation viscose effects, small-scale parameter, Winkler and Pasternak modules, and combined electromechanical loadings are taken into account. The unsteady fluid motion and the effect of viscosity for 


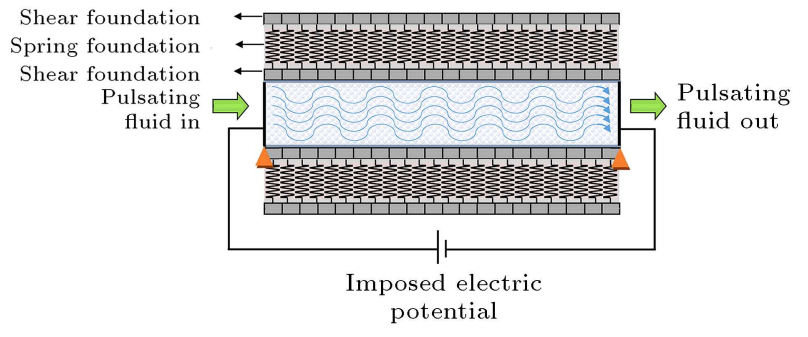

Figure 1. Schematic of a smart embedded micro-shell conveying pulsating fluid.

turbulent flow are modeled using potential flow theory and unsteady time-averaged Navier-Stokes equations, respectively. The governing equations of motion are derived using modal expansion analyses and the modified Lagrangian approach for open systems. In the results section, divergence, flutter, and parametric resonance instabilities are examined in detail. It is concluded that imposing axial electric field may be used as a controlling factor to improve the stability of the system. The results obtained in this study can be particularly utilized to design and manufacture nano/micro electromechanical systems in advanced biomechanics applications with electrical fields as in hydraulic sensors and actuators.

\section{Constitutive equations}

\subsection{Piezoelasticity theory}

Based on piezoelasticity theory, stresses $\{\sigma\}$ and strains $\{\varepsilon\}$, as well as electric displacement $\{D\}$ and electric field $\{E\}$ vectors, in piezoelectric materials, may be combined as follows [35]:

$$
\left\{\begin{array}{c}
\sigma \\
D
\end{array}\right\}=\left[\begin{array}{cc}
C & -e \\
e^{T} & \in
\end{array}\right]\left\{\begin{array}{l}
\varepsilon \\
E
\end{array}\right\}-\left\{\begin{array}{l}
\lambda \\
p
\end{array}\right\} \Delta \Theta,
$$

where the coefficients of thermal expansion, pyroelectric, and temperature change are shown by $\{\lambda\},\{P\}$, and $\Delta \theta$, respectively. Eq. (1) can be expanded in Eq. (2) as shown in Box I; the component of the electric field can be written in terms of scalar functions of electric potential $\phi$ as follows [36]:

$$
E=-\nabla \phi
$$

in which $\nabla$ is the gradient operator.

\subsection{Nonlocal piezoelasticity theory}

Based on the theory of nonlocal elasticity for piezoelectric materials, the stress and electric displacement fields at a reference point $(x)$ of a body are respectively functions of the strain and electrical fields at each point of the body [37-39]. The nonlocal constitutive equations for the piezoelectric material are expressed as follows [40]:

$$
\begin{aligned}
& \sigma_{i j}^{n l}(x)=\int_{V} \alpha\left(\left|x-x^{\prime}\right|, \tau\right) \sigma_{i j}^{l}\left(x^{\prime}\right) d V\left(x^{\prime}\right), \quad \forall x \in V, \\
& (4 \mathrm{a})
\end{aligned}
$$

where $\sigma_{i j}^{n l}$ and $D_{i}^{n l}$ are the components of nonlocal stresses and nonlocal electric displacements, respectively. The kernel function $\alpha\left(\left|x-x^{\prime}\right|, \tau\right)$ is the nonlocal modulus, and $\left|x-x^{\prime}\right|$ is the Euclidean distance. $\tau=$ $e_{0} a / l$ is defined as the scale coefficient that incorporates the small-scale factor, where $e_{0}$ is a constant appropriate for each material and obtained experimentally, and $a$ and $l$ are the internal and external characteristic lengths (e.g., crack length and wavelength), respectively. In addition, $\sigma_{i j}^{l}$ and $D_{i}^{l}$ imply the local stresses and local (i.e., classical) electric displacements obtained from Eq. (2), respectively. According to the axial polarization of the smart micro tube, the nonlocal constitutive equations may be written as follows $[41,42]$ :

$$
\begin{aligned}
& \left(1-\left(e_{0} a\right)^{2} \nabla^{2}\right) \sigma_{i j}^{n l}=\sigma_{i j}^{l}, \\
& \left(1-\left(e_{0} a\right)^{2} \nabla^{2}\right) D_{i j}^{n l}=D_{i j}^{l},
\end{aligned}
$$

$$
\left\{\begin{array}{c}
\sigma_{1} \\
\sigma_{2} \\
\sigma_{3} \\
\tau_{23} \\
\tau_{31} \\
\tau_{12} \\
D_{1} \\
D_{2} \\
D_{3}
\end{array}\right\}=\left\{\begin{array}{ccccccccc}
C_{11} & C_{12} & C_{13} & 0 & 0 & 0 & 0 & 0 & -e_{31} \\
C_{12} & C_{22} & C_{23} & 0 & 0 & 0 & 0 & 0 & -e_{32} \\
C_{13} & C_{23} & C_{33} & 0 & 0 & 0 & 0 & 0 & -e_{33} \\
0 & 0 & 0 & C_{44} & 0 & 0 & 0 & -e_{24} & 0 \\
0 & 0 & 0 & 0 & C_{55} & 0 & -e_{15} & 0 & 0 \\
0 & 0 & 0 & 0 & 0 & C_{66} & 0 & 0 & 0 \\
0 & 0 & 0 & 0 & e_{15} & 0 & \epsilon_{11} & 0 & 0 \\
0 & 0 & 0 & e_{24} & 0 & 0 & 0 & \epsilon_{22} & 0 \\
e_{31} & e_{32} & e_{33} & 0 & 0 & 0 & 0 & 0 & \epsilon_{33}
\end{array}\right\}\left\{\begin{array}{c}
\varepsilon_{1} \\
\varepsilon_{2} \\
\varepsilon_{3} \\
\gamma_{23} \\
\gamma_{31} \\
\gamma_{12} \\
E_{1} \\
E_{2} \\
E_{3}
\end{array}\right\}-\left\{\begin{array}{c}
\lambda_{1} \\
\lambda_{2} \\
\lambda_{3} \\
\lambda_{4} \\
\lambda_{5} \\
\lambda_{6} \\
p_{1} \\
p_{2} \\
p_{3}
\end{array}\right\} \Delta \Theta
$$


where $\nabla^{2}$ represents the Laplace operator. The above implicit relations couple the nonlocal values of stress and electric displacement to their local values. To achieve explicit relations, an iterative-based method can be utilized as follows:

$$
\begin{aligned}
& \left\{\sigma_{i j}^{n l}\right\}^{k+1}=\left(e_{0} a\right)^{2} \nabla^{2}\left\{\sigma_{i j}^{l}\right\}^{k}+\left\{\sigma_{i j}^{l}\right\}, \quad k \geq 0 \\
& \left\{D_{i j}^{n l}\right\}^{k+1}=\left(e_{0} a\right)^{2} \nabla^{2}\left\{D_{i j}^{l}\right\}^{k}+\left\{D_{i j}^{l}\right\}, \quad k \geq 0 \\
& \left\{\sigma_{i j}^{n l}\right\}^{0}=\left\{\sigma_{i j}^{l}\right\} \\
& \left\{D_{i j}^{n l}\right\}^{0}=\left\{D_{i j}^{l}\right\} .
\end{aligned}
$$

In the above iteration loop, the first step is started by the local results and $k$ is the iteration number. Clearly, increasing the iteration number improves the accuracy of the nonlocal results. In addition, the order of the small-scale parameter shows that just a few steps of iteration give enough accuracy, as shown in the numerical results section. From now on, nonlocal components of stresses and electric displacement are used without superscripts.

\subsection{Kinematic relations}

According to nonlinear shallow shell models, the displacement components of an arbitrary point of the body are expressed as follows:

$$
\begin{aligned}
& \tilde{U}(x, \theta, z, t)=u(x, \theta, t)-z \frac{\partial w(x, \theta, t)}{\partial x}, \\
& \tilde{V}(x, \theta, z, t)=v(x, \theta, t)-z \frac{1}{R} \frac{\partial w(x, \theta, t)}{\partial \theta}, \\
& \tilde{W}(x, \theta, z, t)=w(x, \theta, t)
\end{aligned}
$$

where $u, v$ and $w$ are the components of the shell mid-plane displacement along $x, \theta$, and $z$ coordinates, respectively, and $t$ is the time. Based on Donnell's nonlinear shell theory, the strain-displacement relations yield the following:

$$
\left\{\begin{array}{l}
\varepsilon_{x}=\frac{\partial u}{\partial x}+\frac{1}{2}\left(\frac{\partial w}{\partial x}\right)^{2}+z\left(-\frac{\partial^{2} w}{\partial x^{2}}\right) \\
\varepsilon_{\theta}=\frac{1}{R} \frac{\partial \nu}{\partial \theta}+\frac{w}{R}+\frac{1}{2 R^{2}}\left(\frac{\partial w}{\partial \theta}\right)^{2}+z\left(-\frac{1}{R^{2}} \frac{\partial^{2} w}{\partial \theta^{2}}\right) \\
\gamma_{x \theta}=\frac{1}{R} \frac{\partial u}{\partial \theta}+\frac{\partial \nu}{\partial x}+\frac{1}{R} \frac{\partial w}{\partial \theta} \frac{\partial w}{\partial x}+z\left(-\frac{2}{R} \frac{\partial^{2} w}{\partial x \partial \theta}\right)
\end{array}\right.
$$

\subsection{Mode expansion of displacements and electric potential}

By considering simply supported boundary conditions at both ends of the cylindrical shell, the following mode expansion for components of displacement may be considered [43]:

$$
\begin{aligned}
u= & \sum_{m=1}^{M} \sum_{n=0}^{N} \sin \left(\frac{(2 m-1) \pi x}{L}\right)\left[U_{m n}^{c}(t) \cos (n \theta)\right. \\
& \left.+U_{m n}^{s}(t) \sin (n \theta)\right] \\
v= & \sum_{m=1}^{M} \sum_{n=0}^{N} \sin \left(\frac{(2 m-1) \pi x}{L}\right)\left[V_{m n}^{c}(t) \cos (n \theta)\right. \\
& \left.+V_{m n}^{s}(t) \sin (n \theta)\right] \\
w= & \sum_{m=1}^{M} \sum_{n=0}^{N} \sin \left(\frac{(2 m-1) \pi x}{L}\right)\left[W_{m n}^{c}(t) \cos (n \theta)\right. \\
& \left.+W_{m n}^{s}(t) \sin (n \theta)\right]
\end{aligned}
$$

where $m$ and $n$ denote the longitudinal half wave number and circumferential wave number, respectively, $M$ and $N$ are the maximum values of wave numbers. $U_{m n}^{c}(t), U_{m n}^{s}(t), V_{m n}^{c}(t), V_{m n}^{s}(t), W_{m n}^{c}(t)$, and $W_{n m}^{s}(t)$ are time-dependent Degrees Of Free$\operatorname{dom}(\mathrm{DOF})$, and $\left\{q_{d}\right\}=\left[U_{m n}^{c}(t), U_{m n}^{s}(t), V_{m n}^{c}(t)\right.$, $\left.V_{m n}^{s}(t), W_{m n}^{c}(t), W_{m n}^{s}(t)\right]$ is defined as the vector of DOF. The vector dimension of $\left\{q_{d}\right\}$ denotes the number of DOFs, which may be calculated as:

$$
N^{D O F}=3 \times(2 M \times N+M) .
$$

For electric potential, $\phi$, the following mode expansion can be proposed [44]:

$$
\phi=\phi_{0}\left(1-\frac{x}{L}\right)+\sum_{m=1}^{M} \phi_{m}^{s} \sin \left(\frac{(2 m-1) \pi x}{L}\right),
$$

where $\phi_{m}^{s}$ is the time-independent amplitude component of electric potential, $\left\{q_{\phi}\right\}=\left\{\phi_{m}^{s}\right\}$ is defined as the vector of electric potential amplitudes, and $\phi_{0}$ indicates the magnitude of the imposed electric potential. Because of the unidirectional, longitudinally polarized electric field, the electric potential is expanded as a function of the longitudinal coordinate $x$. In the numerical results section, the accuracy of the results will be examined with respect to different values of $M$ and $N$.

In addition, a vector of unknowns can be introduced as follows:

$$
\{q\}=\left\{\left\{q_{d}\right\},\left\{q_{\phi}\right\}\right\}^{T} .
$$




\subsection{Energy functions}

Based on the theory of piezoelectricity, the total potential and kinetic energies of the micro tube with length $L$, cross-section $A$, volume $\forall$, and density $\rho_{s}$ are obtained as follows [45]:

$$
\begin{aligned}
U_{S} & =\frac{1}{2} \int_{0}^{L} \int_{A}\left[\begin{array}{ll}
\sigma & D
\end{array}\right]\left\{\begin{array}{c}
\varepsilon \\
-E
\end{array}\right\} d A d x, \\
T_{S} & =\frac{1}{2} \rho_{S} \iiint_{\forall} \vec{V} \cdot \vec{V} d \forall,
\end{aligned}
$$

where $\sigma$ and $D$ are the nonlocal components of stress and electric displacement and obtained through Eqs. (5). In addition, $\vec{V}$ corresponds to the velocity vector of the micro tube, which leads to the following relation:

$$
T_{S}=\frac{1}{2} \rho_{S} \int_{0}^{L} \int_{A}\left[\left(\frac{\partial u}{\partial t}-z\left(\frac{\partial^{2} w}{\partial x \partial t}\right)\right)^{2}+\left(\frac{\partial w}{\partial t}\right)^{2}\right]
$$

$d A d x$.

The external works done by the surrounded elastic foundation are expressed as follows:

$$
W_{\text {elastic foundation }}=\frac{1}{2} \int_{0}^{L}\left(-K_{W} w+G_{P} \nabla^{2} w\right) w d x
$$

where $K_{W}$ and $G_{P}$ are Winkler's spring foundation modulus and Pasternak's shear foundation modulus, respectively. Moreover, the energies related to the fluid flow are evaluated separately in the following section.

\section{Pulsating fluid flow modeling}

This study considers visco pulsating micro fluid flow inside the micro shell, which is one of the most empirical models. The micro fluid flow is assumed to be turbulent fully developed, Newtonian, irrotational, isentropic, and incompressible. The unsteady fluid motion is obtained based on potential flow theory and, to bring the micro scale problem closer to reality, the effects of pulsating viscosity and slip boundary condition are considered based on unsteady time-averaged NaviesStokes equations and Knudsen number theory [6,10].

Let us assume that no cavitation occurred at the fluid and shell interfaces. An unsteady potential function $\varphi$ may be found, which satisfies the Laplace equation and following boundary condition as [46]:

$$
\begin{aligned}
& \nabla^{2} \varphi=\frac{\partial^{2} \varphi}{\partial x^{2}}+\frac{\partial^{2} \varphi}{\partial r^{2}}+\frac{1}{r} \frac{\partial \varphi}{\partial r}+\frac{1}{r^{2}} \frac{\partial^{2} \varphi}{\partial \theta^{2}}=0 \\
& \left.\frac{\partial \varphi}{\partial r}\right|_{r=R}=\left(\frac{\partial w}{\partial t}+U_{f} \frac{\partial w}{\partial x}\right),
\end{aligned}
$$

where $U_{f}$ is the pulsating flow velocity. By employing the separation of variables method, the general solution of Eq. (16) may be obtained as follows:

$$
\varphi(x, r, \theta, t)=\sum_{m=1}^{M} \sum_{n=0}^{N} \Phi_{m}(x) \Xi_{m, n}(r) \cos (n \theta) f_{m, n}(t) .
$$

By substituting Eq. (18) into (16) besides applying the regularity condition at $r=0$, the unknown functions will be obtained. By utilizing the boundary condition (17), Eq. (18) will be evaluated as follows:

$$
\begin{aligned}
\phi(x, r, \theta, t)= & \sum_{m=1}^{M} \sum_{n=0}^{N} \frac{L}{m \pi} \frac{I_{n}(m \pi r / L)}{I_{n}^{\prime}(m \pi R / L)} \\
& \left(\frac{\partial w_{m, n}}{\partial t}+U_{f} \frac{\partial w_{m, n}}{\partial x}\right),
\end{aligned}
$$

where $I_{n}$ and $I_{n}^{\prime}$ are respectively the modified Bessel function of the first kind of order and its derivative. Consequently, the perturbation pressure at the inner wall of the shell is found as follows [6]:

$$
\begin{gathered}
p=-\rho_{F} \sum_{m=1}^{M} \sum_{n=0}^{N} \frac{L}{m \pi} \frac{I_{n}(m \pi r / L)}{I_{n}^{\prime}(m \pi R / L)} \\
\left(\frac{\partial}{\partial t}+U_{f} \frac{\partial}{\partial x}\right)^{2} w_{m, n},
\end{gathered}
$$

in which $\rho_{F}$ is the density of fluid flow.

\subsection{Potential energy of fluid flow}

Consider the fluid flow inside the micro tube with occupied volume $\Gamma$ and surrounded surface $\Lambda$. The total energy related to the fluid-flow $E_{F}^{T}$ is given by:

$$
E_{F}^{T}=\frac{1}{2} \rho_{f} \iiint_{\Gamma} \vec{V}_{f} \cdot \vec{V}_{f} d \Gamma .
$$

According to Green's theorem, Eq. (21) is evaluated as follows:

$$
E_{F}^{T}=\frac{1}{2} \rho_{f} \iiint_{\Gamma} \nabla \Psi . \nabla \Psi d \Gamma=\frac{1}{2} \rho_{f} \iint_{\Lambda}\left(\Psi \frac{\partial \Psi}{\partial \nu}\right) d \Lambda
$$

where $\Psi$ is the potential function of the fluid flow, and $v$ is the normal vector of boundary surfaces, which is considered positive outward. Integration of Eq. (22) gives:

$$
\begin{aligned}
E_{F}^{T}= & \frac{1}{2} \rho_{f} \int_{0}^{2 \pi} \int_{0}^{L}\left(\Psi \frac{\partial \Psi}{\partial r}\right)_{r=R} d x R d \theta \\
& +\frac{1}{2} \rho_{f} \int_{0}^{2 \pi} \int_{0}^{R}\left(\Psi \frac{\partial \Psi}{\partial x}\right)_{x=L} r d r d \theta \\
& -\frac{1}{2} \rho_{f} \int_{0}^{2 \pi} \int_{0}^{R}\left(\Psi \frac{\partial \Psi}{\partial x}\right)_{x=0} r d r d \theta,
\end{aligned}
$$

in which the boundary conditions are: 


$$
\begin{aligned}
& \frac{\partial \Psi}{\partial r}=\frac{\partial \varphi}{\partial r}, \\
& \frac{\partial \Psi}{\partial x}=U_{f}+\frac{\partial \varphi}{\partial x}, \\
& \left.\Psi\right|_{x=L}=U_{f} L+\left.\varphi\right|_{x=L}, \\
& \left.\Psi\right|_{x=0}=\left.\varphi\right|_{x=L} .
\end{aligned}
$$

By using Eq. (24), Eq. (23) may be obtained as follows:

$$
\begin{aligned}
E_{F}^{T}= & E_{F}^{p}+\frac{1}{2} \rho_{f} \int_{0}^{2 \pi} \int_{0}^{L}\left(U_{f} x \frac{\partial \varphi}{\partial r}\right)_{r=R} d x R d \theta \\
& +\frac{1}{2} \rho_{f} \int_{0}^{2 \pi} \int_{0}^{R}\left(U_{f} L \frac{\partial \varphi}{\partial x}\right)_{x=L} r d r d \theta \\
& +\frac{1}{2} \rho_{f} U_{f}^{2} \pi R^{2} L
\end{aligned}
$$

where $E_{F}^{p}$ denotes the perturbation velocity potential of fluid and is given by:

$$
E_{F}^{p}=\frac{1}{2} \rho_{f} \int_{0}^{2 \pi} \int_{0}^{L}\left(\varphi \frac{\partial \varphi}{\partial r}\right)_{r=R} d x R d \theta .
$$

Moreover, other terms in Eq. (25) have no influence on the total energy of fluid flow. By substituting Eq. (16) into Eq. (17) and employing Eq. (7), we yield the following:

$$
E_{F}^{p}=\frac{1}{2} \rho_{f} \int_{0}^{2 \pi} \int_{0}^{L}(\varphi)_{r=R}\left(\frac{\partial w}{\partial t}+U_{f} \frac{\partial w}{\partial x}\right) d x R d \theta
$$

Using Eq. (9), Eq. (27) may be obtained as follows:

$$
\begin{aligned}
E_{F}^{p}= & \frac{1}{2} \rho_{f} \int_{0}^{2 \pi} \int_{0}^{L} \sum_{m=1}^{M} \sum_{l=1}^{M} \sum_{n=0}^{N} \sum_{k=0}^{N} \frac{L}{m \pi} \frac{I_{n}(m \pi R / L)}{I_{n}^{\prime}(m \pi R / L)} \\
& \times\left(\dot{w}_{m, n}+U_{f} \frac{\partial w_{m, n}}{\partial x}\right)\left(\dot{w}_{l, k}+U_{f} \frac{\partial w_{l, k}}{\partial x}\right) d x R d \theta .
\end{aligned}
$$

By substituting Eq. (28) into Eq. (25), the total energy of fluid is derived and divided as follows:

$$
E_{F}^{T}=T_{F}-V_{F}+E_{G},
$$

where $T_{F}, E_{G}$, and $V_{F}$ are kinetic, gyroscopic, and potential energies of the fluid flow, respectively, and are expressed as follows [6]:

$$
T_{F}=\frac{1}{2} \rho_{f} \sum_{m=1}^{M} \sum_{n=0}^{N} \int_{0}^{2 \pi} \int_{0}^{L} \frac{L}{m \pi} \frac{I_{n}(m \pi R / L)}{I_{n}^{\prime}(m \pi R / L)} \dot{w}_{m, n}^{2}
$$

$d x R d \theta$

$$
\begin{aligned}
V_{F}= & -\frac{1}{2} \rho_{f} \sum_{m=1}^{M} \sum_{n=0}^{N} \int_{0}^{2 \pi} \int_{0}^{L} \frac{L}{m \pi} \frac{I_{n}(m \pi R / L)}{I_{n}^{\prime}(m \pi R / L)} U_{f}^{2} \\
& \left(\frac{\partial w_{m, n}}{\partial x}\right)^{2} d x R d \theta \\
E_{G}= & \frac{1}{2} \rho_{f} \sum_{m=1}^{M} \sum_{l=1}^{M} \sum_{n=0}^{N} \sum_{k=0}^{N} \int_{0}^{2 \pi} \int_{0}^{L} \frac{L}{m \pi} \frac{I_{n}(m \pi R / L)}{I_{n}^{\prime}(m \pi R / L)} U_{f}
\end{aligned}
$$

$$
\left(\dot{w}_{m, n} \frac{\partial w_{l, k}}{\partial x}+\dot{w}_{l, k} \frac{\partial w_{m, n}}{\partial x}\right) d x R d \theta .
$$

The unsteady flow velocity $U_{f}(t)$ for pulsating fluid flow is expressed by the following time-dependent function as follows:

$$
U_{f}(t)=U_{0}(1+\xi \cos (\Omega t)),
$$

where $U_{0}$ is the mean value of flow velocity, $\xi$ is the amplitude of the harmonic fluctuation, and $\Omega$ is its frequency. In addition, it is clear that the expressions of $T_{F}, E_{G}$, and $V_{F}$ will be time dependent in this case and, therefore, this leads to the addition of a new term in the Lagrange equations of motion in Section 4.

\subsection{Viscous effects for pulsatile flow}

In this section, the pulsating fluid viscosity effect is simulated as an external force acting on the inner wall of the shell. Considering a fully developed turbulent and incompressible axial flow regime with fluctuating velocities $\bar{u}_{x}, \bar{u}_{\theta}, \bar{u}_{r}$ in the axial, circumferential, and radial directions, respectively, the time-averaged Navier-Stokes equations are given by:

$$
\begin{aligned}
& \frac{1}{\rho_{f}} \frac{\partial P}{\partial x}=-\frac{1}{r} \frac{d}{d r}\left(r \bar{u}_{x} \bar{u}_{r}\right)+\frac{\eta}{r} \frac{d}{d r}\left(r \frac{d U_{f}}{d r}\right), \\
& \frac{1}{\rho_{f}} \frac{\partial P}{\partial r}=-\frac{1}{r} \frac{d}{d r}\left(r \bar{u}_{r}^{2}\right)+\frac{\bar{u}_{\theta}^{2}}{r}, \\
& 0=\frac{d}{d r}\left(r \bar{u}_{r} \bar{u}_{\theta}\right)+2 \frac{\bar{u}_{r} \bar{u}_{\theta}}{r},
\end{aligned}
$$

where $\eta$ is the kinematic viscosity, and the overbar indicates the time-averaged components. In the following, by using the Computational Fluid Dynamics (CFD), the pressure distribution will be obtained as follows [47]:

$$
\begin{aligned}
P(x, r)= & -2 \frac{\rho_{f}}{R} U_{\tau}^{2} x-\rho_{f} \bar{u}_{r}^{2}+\rho_{f} \int_{R}^{r} \frac{\bar{u}_{\theta}^{2}-\bar{u}_{r}^{2}}{r} d r \\
& +P(0, R),
\end{aligned}
$$

where the stress velocity, $U_{\tau}$, is expressed as follows:

$$
U_{\tau}=\left(-\eta \frac{d U_{f}}{d r}\right)_{r=R}^{1 / 2}=\left(\frac{\tau_{w}}{\rho_{f}}\right)^{1 / 2}=\left(\frac{1}{8} f U_{f}^{2}\right)^{1 / 2},
$$

where $f$ and $\tau_{w}$ are the friction factor and the fluid 
frictional force per unit area of the shell, respectively. The distribution of pressure on the shell interfaces and the drop pressure in the axial direction of the shell are given by:

$$
\begin{gathered}
P(x, R)=-2 \frac{\rho_{f}}{R} U_{\tau}^{2} x+P(0, R) \\
=-\rho_{f}\left(\frac{f}{4 R} U_{f}^{2}+\frac{d U_{f}}{d t}\right) x+P(0, R), \\
\Delta P_{w}=P(0, R)-P(L, R)=\rho_{f}\left(\frac{f}{4 R} U_{f}^{2}+\frac{d U_{f}}{d t}\right) L .
\end{gathered}
$$

By ignoring the effects of flow acceleration, the constant friction traction force in the axial direction is given by:

$$
\tau_{w}=f \rho_{f} U_{f}^{2} / 8 .
$$

Based on the classical fluid mechanic, the friction factor $(f)$ of the micro tube is a function of the Reynolds number $(\mathrm{Re})$ and the mean roughness $(\delta)$ of the inner wall of the shell. The friction factor is estimated through empirical Colebrook equation as follows:

$$
\frac{1}{\sqrt{f}}+2 \log \left(\frac{\delta / 2 R}{3.7}+\frac{2.51}{\operatorname{Re} \sqrt{f}}\right)=0,
$$

in which $\operatorname{Re}=2 R U_{f} / \eta$.

Lastly, the total work associated with the fluid viscosity is given by:

$$
W_{\text {Fluid Viscosity }}=\int_{0}^{2 \pi} \int_{0}^{L}\left(\Delta P_{W}+\tau_{W} u\right) d x R d \theta .
$$

\subsection{Effect of slip boundary conditions}

Often, the FSI problems are assumed with no-slip boundary conditions in the fluid and shell interfaces. However, this assumption will not be valid for the micro-scale problems due to the nonlocality and Knudsen number effects [48]. In other words, based on Kn theory, the value of $\mathrm{Kn}$ in the micro-scale problems will exceed 0.01 and, therefore, an average fluid Velocity Correction Factor (VCF) should be applied to all of the following equations as follows [49]:

$$
V C F=\frac{V_{a v g, \text { slip }}}{V_{a v g, n o-s l i p}}=\left(4\left(\frac{2-\sigma_{v}}{\sigma_{v}}\right)\left(\frac{\mathrm{Kn}}{1+\mathrm{Kn}}\right)+1\right),
$$

where $\sigma_{v}$ is the tangential momentum accommodation coefficient and is measured to be 0.7 for most practical purposes [50].

\section{Method of solution}

This study utilized the modified Lagrange equations of motion for open systems with a control volume consisting of the micro shell and the fluid flow passing through it $[27,51]$ :

$$
\begin{gathered}
\frac{d}{d t}\left[\frac{\partial\left(T_{S}+T_{F}+E_{G}\right)}{\partial \dot{q}_{k}}\right]-\frac{\partial\left(T_{S}+T_{F}+E_{G}\right)}{\partial q_{k}} \\
\quad+\frac{\partial\left(U_{S}+V_{F}\right)}{\partial q_{k}}=Q_{k}, \quad k=1, \ldots, 3 N,
\end{gathered}
$$

in which $q_{k}$ is the element of vector $\{q\}$ previously introduced in Section 2.5, the terms $\frac{\partial T_{S}}{\partial q_{k}}$ and $\frac{\partial T_{F}}{\partial q_{k}}$ are zero, and $Q_{k}$ is the generalized force obtained by differentiation of the virtual work done through external forces:

$$
Q_{k}=\frac{\partial W}{\partial q_{k}}
$$

By employing mode expansions (9) and (10), Eq. (43) is evaluated as follows:

$$
[M]\{\ddot{q}\}+[C(t)]\{\dot{q}\}+[K(t)]\{q\}=\{Q\},
$$

where $[M]$ and $[C(t)]$ are the mass and damping matrices, respectively, and $[K(t)]$ is the stiffness matrix composed of linear and nonlinear terms as follows:

$$
[K(t)]=\left[K_{L}(t)+K_{N L}(t)\right]
$$

Because of static coupling between mechanical and electric displacement, Eq. (45) may be rearranged based on Eq. (11) as follows:

$$
\begin{gathered}
{\left[\begin{array}{cc}
M_{d d} & 0 \\
0 & 0
\end{array}\right]\left\{\begin{array}{l}
\ddot{q}_{d} \\
\ddot{q}_{\phi}
\end{array}\right\}+\left[\begin{array}{cc}
C_{d d} & 0 \\
0 & 0
\end{array}\right]\left\{\begin{array}{l}
\dot{q}_{d} \\
\dot{q}_{\phi}
\end{array}\right\}} \\
+\left[\begin{array}{ll}
K_{d d} & K_{d \phi} \\
K_{\phi d} & K_{\phi \phi}
\end{array}\right]\left\{\begin{array}{l}
q_{d} \\
q_{\phi}
\end{array}\right\}=\left\{\begin{array}{l}
Q_{d} \\
Q_{\phi}
\end{array}\right\} .
\end{gathered}
$$

According to the lack of external electrical load, the second sets of Eq. (47) lead to the following:

$$
\left\{q_{\phi}\right\}=-\left[K_{\phi \phi}^{-1} K_{\phi d}\right]\left\{q_{d}\right\} .
$$

Through Eq. (48), $q_{\phi}$ in the first sets of Eq. (47) is eliminated and yields a set of modified equations of motion as:

$$
\left[M_{d d}\right]\left\{\ddot{q}_{d}\right\}+\left[C_{d d}(t)\right]\left\{\dot{q}_{d}\right\}+\left[K_{M}(t)\right]\left\{q_{d}\right\}=\left\{Q_{M}\right\},
$$

where:

$$
\begin{aligned}
& K_{M}=K_{d d}-K_{d \phi} K_{\phi \phi}^{-1} K_{\phi d}, \\
& Q_{M}=Q_{d}-K_{d \phi} K_{\phi \phi}^{-1} Q_{\phi} .
\end{aligned}
$$

Of note, the nonlinear modified stiffness matrix $\left[K_{M}(t)\right]$ and the damping matrix $\left[C_{d d}(t)\right]$ in Eq. (49) are harmonically time dependent by considering the pulsatile flow regime according to Eq. (34) as follows:

$$
\begin{aligned}
& K_{M}(t)=f_{1}(\sin \Omega t, \cos \Omega t), \\
& C_{d d}(t)=f_{2}(\sin \Omega t, \cos \Omega t) .
\end{aligned}
$$




\subsection{Dynamic instability formulation}

For static fluid velocity condition $(\xi=0)$, the following general solution can be expressed for Eq. (49) as follows:

$$
\left\{q_{d}\right\}=\left\{\hat{q}_{d}\right\} \exp (\omega t),
$$

in which $\left\{\hat{q}_{d}\right\}$ is the amplitude of the displacements vector, and $\omega$ is a complex circular frequency composed of real and imaginary parts that represent damping and natural frequencies, respectively.

By introducing the secondary vector $\{S\}=$ $\omega\left\{q_{d}\right\}$, Eq. (49) is converted to the following state space problem:

$$
[\Psi]\{X\}=\omega\{X\},
$$

where:

$$
\begin{aligned}
& {[\Psi]=\left[\begin{array}{cc}
0 & \mathrm{I} \\
-M_{d d}^{-1} K_{M} & -M_{d d}^{-1} C_{d d}
\end{array}\right],} \\
& \{X\}=\left[\left\{q_{d}\right\},\{S\}\right]^{T} .
\end{aligned}
$$

Eq. (53) is an eigenvalue problem with nonlinear stiffness terms that can be solved according to the following iterative procedure [52]:

Step 1: Calculating linear eigenvalues and eigenvectors by ignoring all of the nonlinear terms in the stiffness matrix;

Step 2: Scaling up the eigenvectors and estimating nonlinear terms in the stiffness matrix;

Step 3: Considering nonlinear and linear components of the stiffness matrix together and evaluating eigenvectors and eigenvalues of the updated eigenvalue problem;

Step 4: Comparing new and previous eigenvectors until reaching the convergence conditions; otherwise, Steps 2 to 4 must be repeated.

In this study, the above procedure is performed until the maximum relative error between the two successive iterations becomes less than $0.1 \%$.

\subsection{Parametric instability formulation}

In order to study the parametric instability of the system, it is usual to study the effects of amplitude $(\xi)$ and frequency $(\Omega)$ of the pulsating flow on the system stability at various static fluid velocity $U_{0}$ levels. The boundaries of the regions of parametric resonance may be obtained usually via the Bolotin's [53] or Floquet methods. Based on Bolotin's method, the generalized coordinate is assumed to be periodic and is expressed in the following form:

$$
q_{d}(t)=\sum_{k=0}^{\bar{N}}\left\{a_{k} \sin \left(\frac{k \Omega t}{2}\right)+b_{k} \cos \left(\frac{k \Omega t}{2}\right)\right\} .
$$

By substituting Eq. (55) into Eq. (49) and setting the coefficients of harmonics to zero, the following algebraic equation is obtained:

$$
[C]\{Y\}=\{0\},
$$

where $\{Y\}=\left\{b_{0}, a_{1}, b_{1}, \ldots, a_{\bar{N}}, b_{\bar{N}}\right\}^{T}$ is the amplitude vector. The characteristic equation of this problem is expressed as follows:

$$
f(C)=\operatorname{det}(C)=0 .
$$

This equation is a nonlinear algebraic equation on $\Omega$, which is solved numerically by the mathematical software to detect the system's parametric resonance instability boundaries.

\section{Numerical results}

In this section, the dynamical instabilities of a smart micro tube surrounded by an elastic foundation and under internal pulsating micro flow and axial electric field are examined, as shown in Figure 1. To this end, divergence and flutter instabilities, which occur by increasing the mean flow velocity $\left(U_{0}\right)$, and parametric resonance instability in the case of increasing dimensionless pulsation amplitude $(\xi)$ are studied. The micro tube is considered to be made of piezoelectric materials as PZT4 and its structural properties are presented in Table 1. A comprehensive parametric study has been made based on the effects of fluid flow components (mean flow velocity and the frequency and pulsating magnitudes), small-scale parameter, elastic foundation modules, Knudsen number, fluid viscosity, geometric nonlinearity, and applied electric voltage.

\subsection{Accuracy of the results}

The accuracy of the results is examined in Table 2 for different magnitudes of $M$ and $N$ and for both linear and nonlinear approaches. From this table, it can be

Table 1. Properties of PZT4.

\begin{tabular}{cc}
\hline Properties of PZT4 & Ref. [45] \\
\hline$C_{11}(\mathrm{GPa})$ & 139 \\
$C_{22}(\mathrm{GPa})$ & 115 \\
$C_{12}(\mathrm{GPa})$ & 77.8 \\
$e_{11}\left(\mathrm{C} / \mathrm{m}^{2}\right)$ & 15.1 \\
$e_{12}\left(\mathrm{C} / \mathrm{m}^{2}\right)$ & -5.2 \\
$\epsilon_{11}\left(\mathrm{C}^{2} / \mathrm{Nm}^{2}\right)$ & $6.46 \times 10^{-9}$ \\
$p_{11}\left(\mathrm{C} / \mathrm{m}^{2} \mathrm{~K}\right)$ & $-2.5 \times 10^{-} 5$ \\
$\alpha_{1}(1 / \mathrm{K})$ & $7.41 \times 10^{-6}$ \\
$\alpha_{2}(1 / \mathrm{K})$ & $2.11 \times 10^{-6}$ \\
$\rho\left(\mathrm{kg} / \mathrm{m}^{3}\right)$ & 7500 \\
$L / R$ & 20 \\
\hline
\end{tabular}


Table 2. Accuracy of the results for various mode expansions.

\begin{tabular}{|c|c|c|c|c|c|c|}
\hline \multirow[b]{2}{*}{ s } & \multicolumn{3}{|c|}{$N\left(U_{0}^{*}=0.0001, \xi=0\right)$} & \multicolumn{3}{|c|}{$N\left(U_{0}^{*}=0.001, \xi=0\right)$} \\
\hline & 1 & 2 & 3 & 1 & 2 & 3 \\
\hline & $N^{D O F}=9$ & $N^{D O F}=15$ & $N^{D O F}=21$ & $N^{D O F}=9$ & $N^{D O F}=15$ & $N^{D O F}=21$ \\
\hline 1 & $\operatorname{Im}\left(\omega^{*}\right)_{N L}=0.00934$ & $\operatorname{Im}\left(\omega^{*}\right)_{N L}=0.00942$ & $\operatorname{Im}\left(\omega^{*}\right)_{N L}=0.00947$ & $\operatorname{Im}\left(\omega^{*}\right)_{N L}=0.00498$ & $\operatorname{Im}\left(\omega^{*}\right)_{N L}=0.00523$ & $\operatorname{Im}\left(\omega^{*}\right)_{N L}=0.00537$ \\
\hline & $\operatorname{Im}\left(\omega^{*}\right)_{L}=0.00925$ & $\operatorname{Im}\left(\omega^{*}\right)_{L}=0.00925$ & $\operatorname{Im}\left(\omega^{*}\right)_{L}=0.00925$ & $\operatorname{Im}\left(\omega^{*}\right)_{L}=0.00460$ & $\operatorname{Im}\left(\omega^{*}\right)_{L}=0.00460$ & $\operatorname{Im}\left(\omega^{*}\right)_{L}=0.00460$ \\
\hline & $N^{D O F}=18$ & $N^{D O F}=30$ & $N^{D O F}=42$ & $N^{D O F}=18$ & $N^{D O F}=30$ & $N^{D O F}=42$ \\
\hline 2 & $\operatorname{Im}\left(\omega^{*}\right)_{N L}=0.00943$ & $\operatorname{Im}\left(\omega^{*}\right)_{N L}=0.00946$ & $\operatorname{Im}\left(\omega^{*}\right)_{N L}=0.00950$ & $\operatorname{Im}\left(\omega^{*}\right)_{N L}=0.00531$ & $\operatorname{Im}\left(\omega^{*}\right)_{N L}=0.00542$ & $\operatorname{Im}\left(\omega^{*}\right)_{N L}=0.00548$ \\
\hline & $\operatorname{Im}\left(\omega^{*}\right)_{L}=0.00925$ & $\operatorname{Im}\left(\omega^{*}\right)_{L}=0.00925$ & $\operatorname{Im}\left(\omega^{*}\right)_{L}=0.00925$ & $\operatorname{Im}\left(\omega^{*}\right)_{L}=0.00460$ & $\operatorname{Im}\left(\omega^{*}\right)_{L}=0.00460$ & $\operatorname{Im}\left(\omega^{*}\right)_{L}=0.00460$ \\
\hline & $N^{D O F}=27$ & $N^{D O F}=45$ & $N^{D O F}=63$ & $N^{D O F}=27$ & $N^{D O F}=45$ & $N^{D O F}=63$ \\
\hline 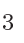 & $\operatorname{Im}\left(\omega^{*}\right)_{N L}=0.00950$ & $\operatorname{Im}\left(\omega^{*}\right)_{N L}=0.00950$ & $\operatorname{Im}\left(\omega^{*}\right)_{N L}=0.00950$ & $\operatorname{Im}\left(\omega^{*}\right)_{N L}=0.00550$ & $\operatorname{Im}\left(\omega^{*}\right)_{N L}=0.00552$ & $\operatorname{Im}\left(\omega^{*}\right)_{N L}=0.00552$ \\
\hline & $\operatorname{Im}\left(\omega^{*}\right)_{L}=0.00925$ & $\operatorname{Im}\left(\omega^{*}\right)_{L}=0.00925$ & $\operatorname{Im}\left(\omega^{*}\right)_{L}=0.00925$ & $\operatorname{Im}\left(\omega^{*}\right)_{L}=0.00460$ & $\operatorname{Im}\left(\omega^{*}\right)_{L}=0.00460$ & $\operatorname{Im}\left(\omega^{*}\right)_{L}=0.00460$ \\
\hline
\end{tabular}

concluded that the accuracy of the results is sufficiently enough for linear analyses if $(M, N)=(1,1)$ (i.e., DOF $=9)$ and for nonlinear analyses if $(M, N)=(3,1)$ (i.e., $\mathrm{DOF}=27)$.

\subsection{Validation of the results}

In order to validate the present results, at first, the piezoelectric properties of the structure, small-scale parameters, and elastic medium are neglected, and the fluid flow velocity is assumed constant. The shell specifications used in this simulation include:

$$
\begin{array}{ll}
R=0.041275 \mathrm{~m}, & L=0.1206 \mathrm{~m}, \\
h=0.127 \mathrm{~mm}, & E=70 \times 10^{9} \mathrm{~Pa}, \\
\rho=2700 \mathrm{~kg} / \mathrm{m}^{3}, & \rho_{F}=1000 \mathrm{~kg} / \mathrm{m}^{3}, \quad \nu=0.33 .
\end{array}
$$

However, the results are compared with the findings of Amabili and Graziera [54], as shown in Figure 2, for vibration and stability of the shell conveying fluid. It can be seen that the obtained results are close to those reported by Amabili and Graziera [54], demonstrating the validity of this work.

For another comparison, the piezoelectric properties of the structure and elastic medium are neglected. In other words, the vibration of a cylindrical shell considering size effects is studied considering:

$$
\begin{array}{ll}
h=0.34 \mathrm{~mm}, & E=1.6 T \mathrm{~Pa}, \\
D=0.678 \mathrm{~nm}, \quad \nu & =0.19 .
\end{array}
$$

Table 3 shows the frequency of the structure for different length-to-diameter ratios. It is found that the

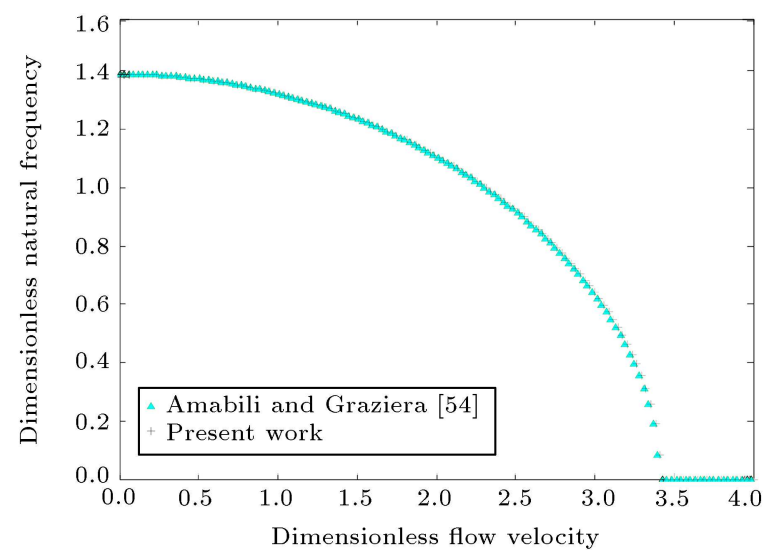

Figure 2. Comparison of the dimensionless natural frequency and dimensionless flow velocity for the simplified analyses of the present work and Amabili and Graziera [54].

results of this paper are in good agreement with the data of Mohammadi et al. [55].

Now, let us introduce the dimensionless parameters used in this study as follows:

$$
\begin{aligned}
& U_{0}^{*}=U_{0} \sqrt{\rho_{f} / C_{11}}, \quad \Omega^{*}=\Omega L \sqrt{\rho / C_{11}}, \\
& \omega^{*}=\omega L \sqrt{\rho / C_{11}}, \quad \eta=\frac{e_{0} a}{L}, \quad \mu^{*}=\frac{\mu A_{f}}{\sqrt{E I m_{f}}}, \\
& K_{w}^{*}=K_{w} / C_{11}, \quad G_{P}^{*}=G_{P} / C_{11} R h, \quad \bar{W}=\frac{w}{L}, \\
& \bar{X}=\frac{x}{L} .
\end{aligned}
$$

The results will be presented in the dimensionless form.

Table 3. Comparison of the natural frequencies (THz) obtained by the nonlocal elasticity theory.

\begin{tabular}{cccc}
\hline $\boldsymbol{L} / \boldsymbol{D}$ & Mohammadi et al. [55] & Present work & Error (\%) \\
\hline 4.86 & 0.776 & 0.768 & 1.03 \\
7.47 & 0.346 & 0.341 & 1.44 \\
13.89 & 0.161 & 0.158 & 1.86 \\
17.47 & 0.114 & 0.111 & 2.63 \\
\hline
\end{tabular}




\subsection{Modal analyses and critical flow velocities for static fluid flow $(\xi=0)$}

Effects of increasing $U_{0}^{*}$ on the natural and damping frequencies of the smart micro tube for the first three vibrational modes are studied in Figures $3(\mathrm{a})$ and 3 (b). As can be seen from these figures, by increasing $U_{0}^{*}$, the imaginary part of natural frequencies will decrease until reaching the value zero at $U_{0}^{*}=0.0012$, 0.0023 , and 0.0048 (for the first, second, and third modes, respectively), which are introduced as the critical flow velocities. It is clear from Figure $3(\mathrm{~b})$ that, at these points, the real part of frequencies becomes nonzero and divergence instability will occur. For more clarity, let us consider the increasing flow velocity for the first mode (fundamental mode). In this mode, at first, the imaginary part of frequency decreases by increasing flow velocity, which implies less stable conditions. The critical flow velocity will be $U_{0}^{*}=0.0012$, and the divergence instability occurs via a pitchfork bifurcation point, as shown in Figure $3(\mathrm{~b})$. In addition, by increasing flow velocities to a greater extent, it becomes clear that in the range

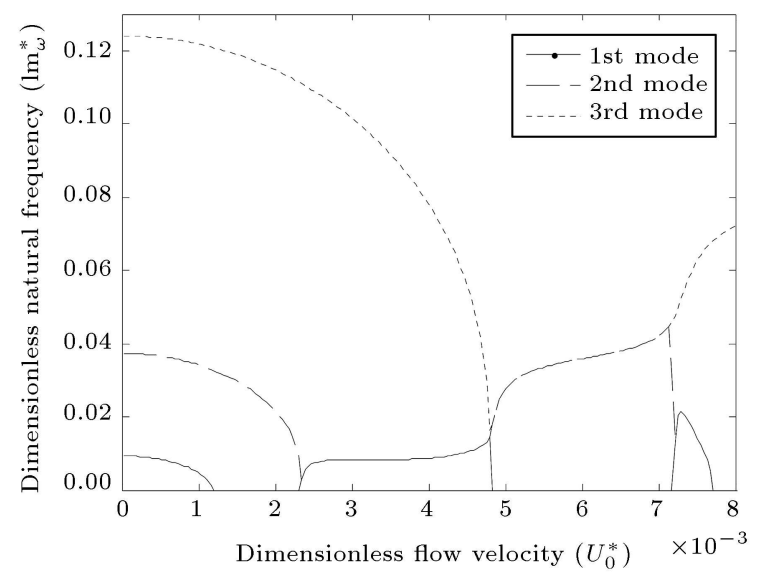

Figure 3(a). Dimensionless natural frequencies versus dimensionless flow velocity for the 1 st to 3 rd modes.

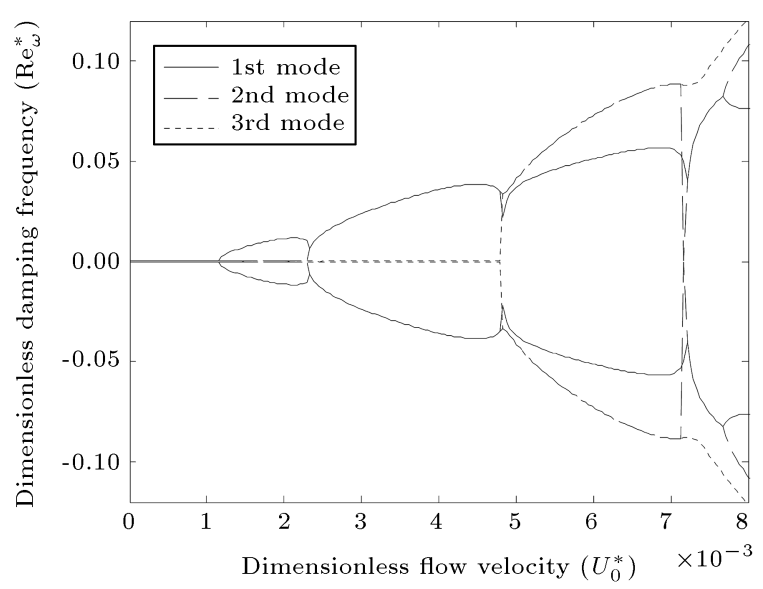

Figure 3(b). Dimensionless damping frequencies versus dimensionless fluid velocity for the 1 st to 3 rd modes. of $0.0012<U_{0}^{*}<0.0048$, the first and second modes will merge, which is another kind of instability known as flutter instability. Flutter instability occurs more often at higher flow velocities. Figure 4. depicts the effects of the small-scale parameter on the fundamental frequency and the critical flow velocity. It is clear that the small-scale parameter should be considered in the order of structural characteristic length of the system. Therefore, in this study, a maximum value of about $120 \mathrm{~nm}$ on a small scale is considered. According to this figure, by increasing flow velocity, the frequency and the critical flow velocity will decrease at an increasing rate. The variation of fluid viscosity magnitude is demonstrated in Figure 5. It can be observed that the influence of viscosity becomes more remarkable when the fluid velocity increases. Additionally, the fundamental frequency and the critical flow velocity of the system will increase when the viscosity of fluid increases, which means that the stability of the system has improved. Effects of externally applied voltage on the fundamental frequency are shown in Figure 6. It

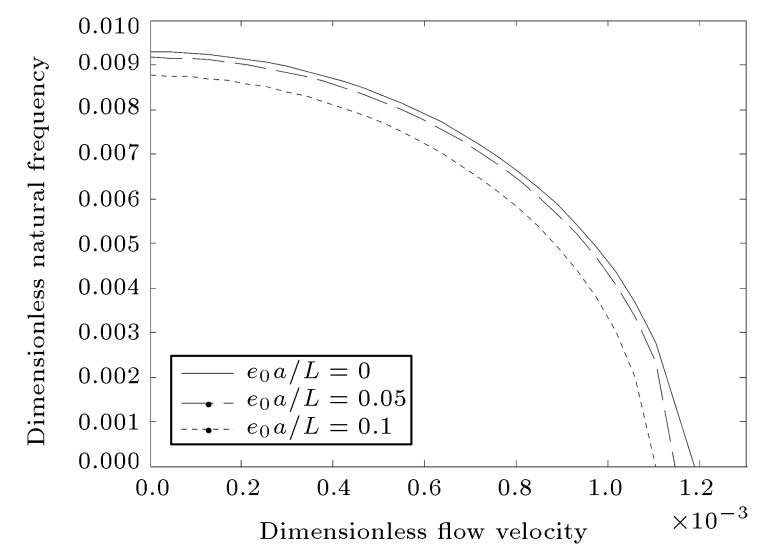

Figure 4. Dimensionless flow velocity versus dimensionless natural frequency for various small-scale parameters for $\xi=0$.

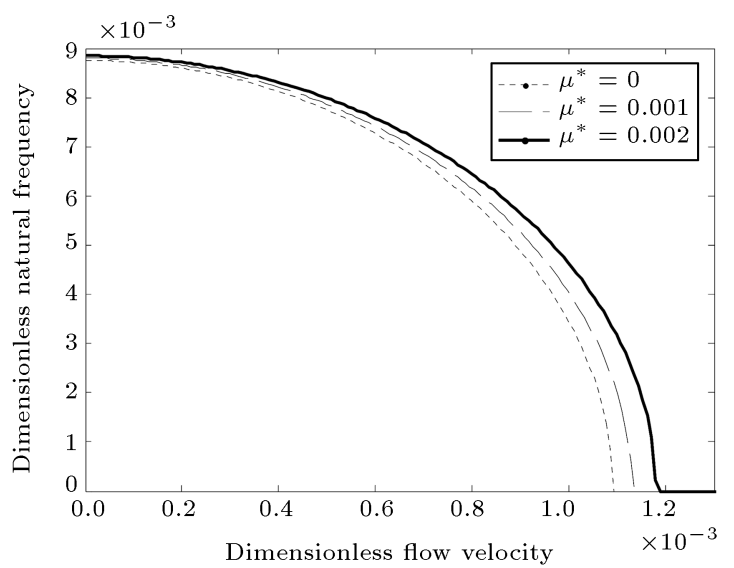

Figure 5. Dimensionless flow velocity versus dimensionless natural frequency for various magnitude of fluid viscosity for $\xi=0$. 
is concluded that, at a constant flow velocity, applying positive (direct) electric potential increases the fundamental frequency and the critical flow velocity, which means that the stability of the system has increased. It is also found that imposing negative (indirect) electric potential decreases the fundamental frequency of the system. This statement means that the application of external electric field is an effective controlling parameter for the smart fluid-conveying systems. To compare the magnitudes of linear and nonlinear natural frequencies, Figure 7 shows the variations of frequency ratio versus mean flow velocity for different small-scale parameters. As can be seen from this figure, when the mean flow velocity is lower than $U_{0}^{*}<0.0004$, the difference between nonlinear and linear frequencies is lower than $3 \%$ for static fluid flow $(\xi=0)$ and is lower than $5 \%$ for dynamic fluid flow $(\xi=0.02)$. It can be concluded that the effects of geometric nonlinearity will increase in case of an increase in the velocity of fluid flow, and this is more remarkable when the fluid flow is dynamic. Effects of the small-scale parameter are also

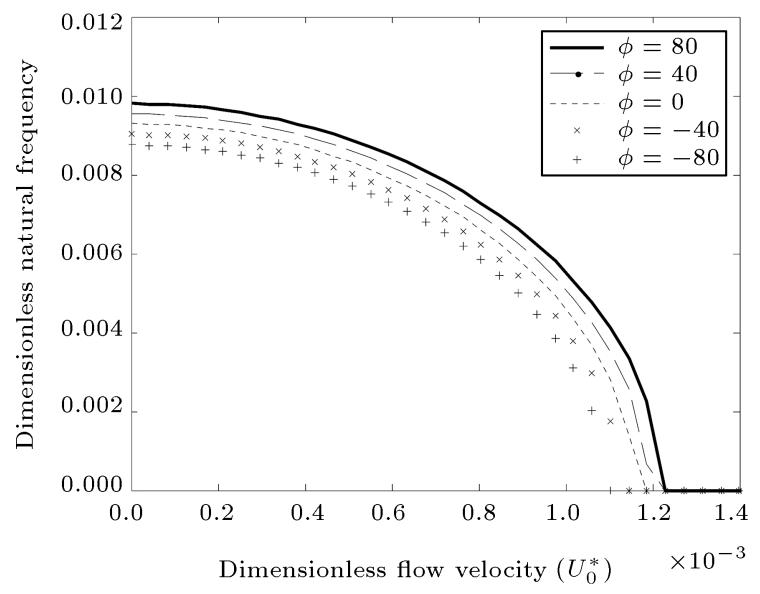

Figure 6. Dimensionless flow velocity versus dimensionless natural frequency for various applied voltages for $\xi=0$.

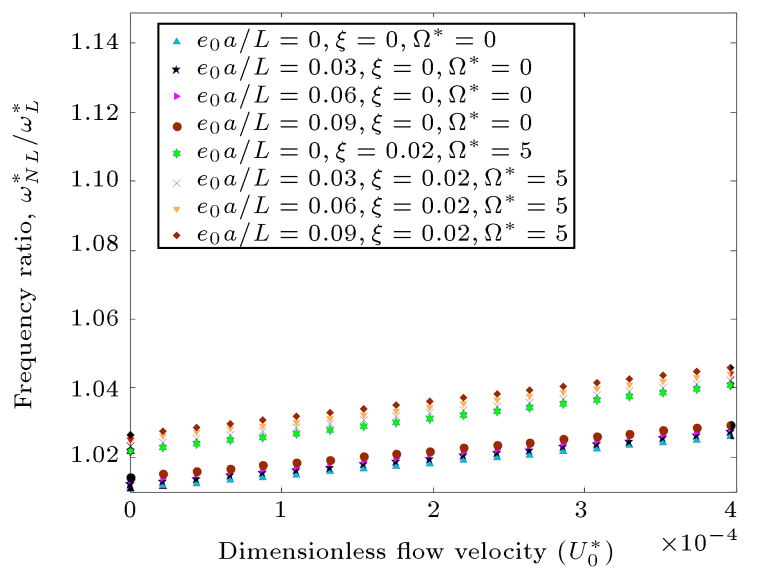

Figure 7. Frequency ratio versus dimensionless flow velocity for different values of the small-scale parameter. considered in this figure. It is shown that considering the small-scale parameter increases the frequency ratio of the system for both dynamic and static fluid flows.

\subsection{Parametric instability of dynamic fluid flow $(\xi \neq 0)$}

By enhancing the dimensionless pulsation amplitude of fluid flow $(\xi)$, the parametric resonance instability occurs, and its boundary curves are achieved through Eq. (57). According to previous literatures, the results of this analysis will be commonly examined in the frequency, $\Omega$, amplitude, $\eta$, plane at various fixed levels of all other influencing parameters such as mean flow velocity, viscosity, small-scale parameter, etc. Figures 8 to 12 show the instability boundary curves for the system. From all of these figures, the regions outside and inside the curves are related to the stable and unstable conditions, respectively. According to these figures, by increasing the mean flow velocity, the instability boundaries move to the down and left sides and will expand beyond the previous ones. This means that a smaller pulsating amplitude and a wider range of pulsating frequencies lead to instability.

Effects of the small-scale parameter are particularly studied in Figure 8. As can be seen from this figure, the effects of nonlocality are more significant at higher flow velocities. It is shown that, given the nonlocality affects, the resonance regions move from higher frequencies to lower ones (towards left and slightly down). This means that the natural frequencies of the system have decreased. In addition, at a constant $(\xi)$, the resonance region will be narrower than previous one, which means also a more stable condition in this case. Figure 9 shows the instability boundaries of the viscous-fluid conveying beam. It is observed that the effects of fluid viscosity strongly depend on the mean flow velocity. As the mean flow velocity increases, the influences of the fluid viscosity become more significant. Additionally, with an increase

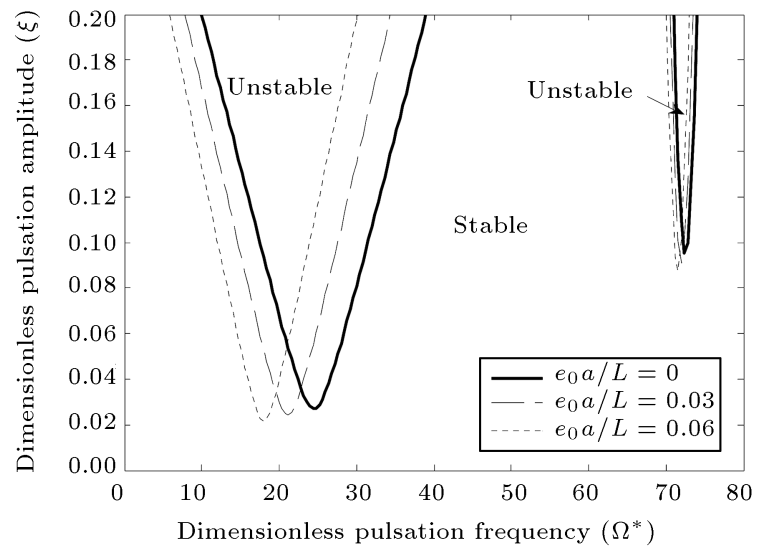

Figure 8. Instability region of a smart micro shell for $U_{0}^{*}=0.0001$ (right side) and $U_{0}^{*}=0.001$ (left side) and various dimensionless small-scale parameters. 


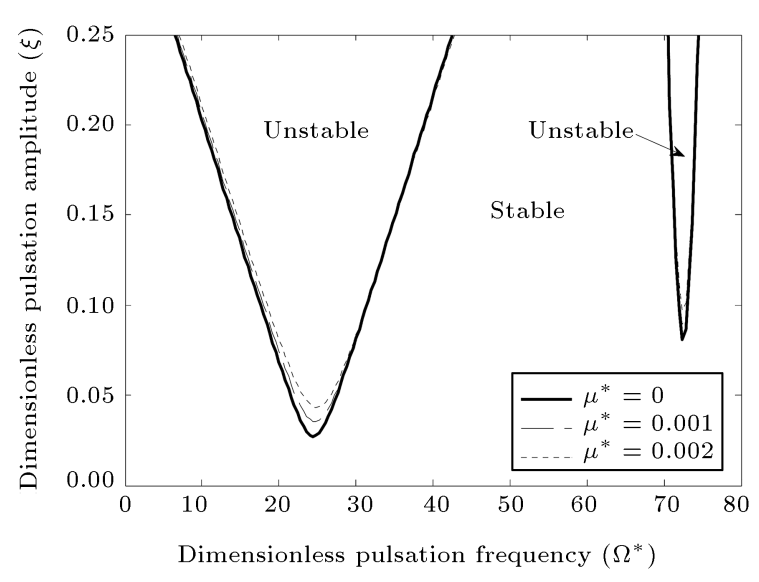

Figure 9. Instability boundaries of a smart micro shell for $U_{0}^{*}=0.0001$ (right side) and $U_{0}^{*}=0.001$ (left side) and various dimensionless fluid viscosities.

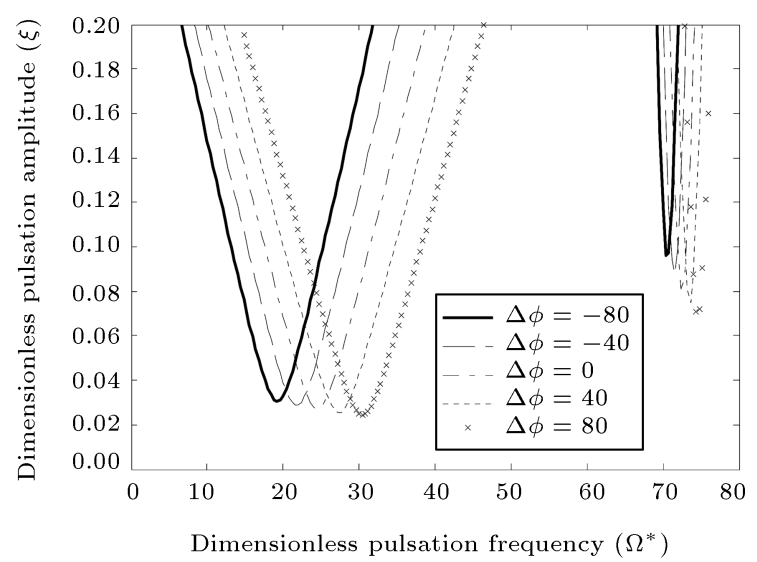

Figure 10. Effects of applied electric field on instability boundaries of a smart micro shell for $U_{0}^{*}=0.0001$ (right side) and $U_{0}^{*}=0.001$ (left side).

in fluid viscosity, the area of the instability region reduces and the instability boundaries slightly shift to the right. This is because of the increasing natural frequencies of the system, as observed in Figure 6. To evaluate the effects of the piezoelectric properties of the smart shell, the instability response of the system is obtained by applying direct and indirect external voltages. Figure 10 illustrates the instability boundaries of the system under different magnitudes of the applied voltage. It is found that by applying direct voltage, the instability curves move to the right, which means that the resonance frequencies of the system have increased. In other words, direct voltage increases the stability of the system and indirect voltage does the opposite. Figure 11 illustrates the effects of both Winkler and Pasternak elastic foundations on the instability boundaries of the system for $U_{0}^{*}=0.001$. It is shown that the unstable areas of the system shift to the left when the elastic foundation exists, because the stiffness of the elastic foundation absorbs the vibrating energy of the system and makes a stiffer and more stable structure.

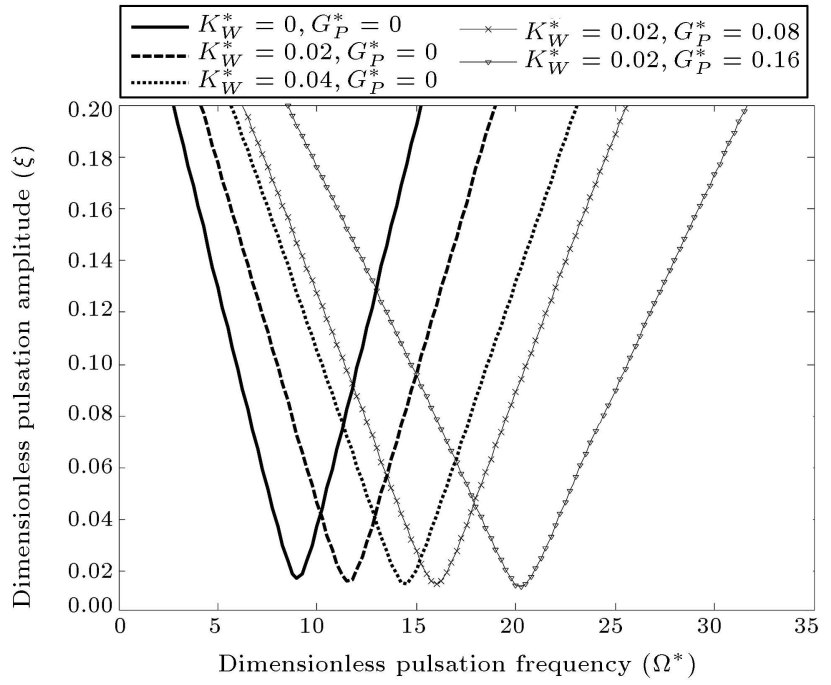

Figure 11. Effects of elastic medium on instability boundaries of a smart micro shell for $U_{0}^{*}=0.001$.

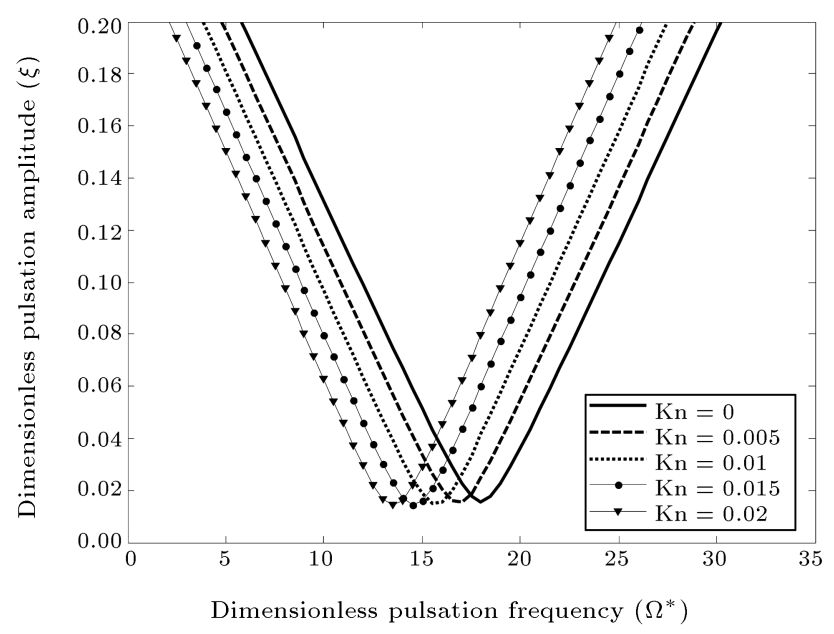

Figure 12. Effects of Kn on instability boundaries of a smart micro shell for $U_{0}^{*}=0.001$.

Figure 11 depicts a more stable condition from the Pasternak foundation (considering both normal and shear effects) than the Winkler foundation with only normal loads. For a micro fluid flow, the value of $\mathrm{Kn}$ is in the range of $10^{-2}<\mathrm{Kn}<10^{-1}$ and, therefore, the slippery regime occurs. It can be seen from Figure 12 that the enhancement of Kn value moves the instability area to the right and down, which means a less stable condition. In fact, increasing Kn means increasing the mean free path of fluid and results in the lower stiffness of the system.

\section{Conclusion}

Instability prediction of a fluid-conveying pipe (as a historical challenge for the engineers) on a micro scale with an internal pulsating flow regime was investigated in this study. To obtain the stability active control of 
the system (sensing and actuating), the piezoelectric material was employed for the body, and effects of applying external electric field on the stability of the system were investigated. Nonlocal piezoelasticity theory, nonlinear cylindrical shell model, and energy approaches were employed to obtain the nonlinear electro statically coupled equations of motion. By applying mode expansion analyses, the equations of motion were discretized and solved via the state space problem. Consequently, the boundaries of the parametric resonance instability were achieved via Bolitin's method. In the numerical results, effects of various parameters such as mean flow velocity, small-scale parameter, applied voltage, fluid viscosity, Knudsen number, and elastic foundation constants on dynamical instabilities of the system were studied. It was shown that the imposed positive electric potential field along the micro shell increased natural frequency, critical flow velocity, and the stability of the structure and vice versa. In addition, it was observed that the fluid viscosity and small-scale parameter had major effects on the critical flow velocity and the stability of the system. This work was presented to extend or complete the application of hydraulic sensors or actuators in advanced nano/micro electro-mechanical systems.

\section{References}

1. Gao, J. and $\mathrm{Xu}, \mathrm{B}$. "Applications of nanomaterials inside cells", Nano Today, 4(1), pp. 37-51 (2009).

2. Kong, J., Franklin, N.R., Zhou, C., Chapline, M.G., Peng, S., Cho, K., and Dai, H. "Nanotube molecular wires as chemical sensors", Science, 287(5453), pp. 622-625 (2000).

3. Dharap, P., Li, Z., Nagarajaiah, S., and Barrera, E.V. "Nanotube film based on single-wall carbon nanotubes for strain sensing", Nanotechnology, 15(3), p. 379 (2004).

4. Ashley, H. and Haviland, G. "Bending vibrations of a pipeline containing flowing fluid", J. Appl. Mech., 72(1), pp. 229-232 (1950).

5. Paidoussis, M.P., Fluid-Structure Interactions: Slender Structures and Axial Flow, 1, Academic Press, London, England (1998).

6. Amabili, M., Nonlinear Vibrations and Stability of Shells and Plates, Cambridge University Press, Parma, Italy (2008).

7. Reddy, J.N. and Wang, C.M., Dynamics of Fluid Conveying Beams: Governing Equations and Finite Element Models, Centre for Offshore Research and Engineering National University of Singapore (2004).

8. Pellicano, F. and Amabili, M. "Dynamic instability and chaos of empty and fluid-filled circular cylindrical shells under periodic axial loads", J. Sound Vib., 293(1), pp. 227-252 (2006).
9. Sadeghi, M.H. and Karimi-Dona, M.H. "Dynamic behavior of a fluid conveying pipe subjected to a moving sprung mass: an FEM-state space approach", Int. J. Press Vessels Pip., 88, pp. 31-123 (2011).

10. Gu, J., Ma, T., and Menglan, D. "Effect of aspect ratio on the dynamic response of a fluid-conveying pipe using the Timoshenko beam model", Ocean Eng, 114, pp. 185-191 (2016).

11. Kamm, R.D. and Pedley, T.J. "Flow in collapsible tubes: a brief review", J. Biomech. Eng., 111, pp. 177-179 (1989).

12. Paidoussis, M.P., Fluid-Structure Interactions: Slender Structures and Axial Flow, 2, Academic Press (2003).

13. Yan, Y., Wang, W.Q., and Zhang, L.X. "Dynamical behaviors of fluid-conveyed multi walled carbon nanotubes", Appl. Math. Modell., 33, pp. 1430-1440 (2009).

14. Kuang, Y.D., He, X.Q., Chen, C.Y., and Li, G.Q. "Analysis of nonlinear vibrations of double-walled carbon nanotubes conveying fluid", Int. J. Comput. Mater. Sci. Surf. Eng., 45, pp. 875-880 (2009).

15. Ghorbanpour Arani, A., Shajari, A.R., Amir, S., and Atabakhshian, V. "Nonlinear fluid-induced vibration and instability of an embedded piezoelectric polymeric microtube using nonlocal elasticity theory", J. Mech. Eng. Sci., 227(12), pp. 2870-2885 (2013).

16. Ghorbanpour Arani, A., Shajari, A.R., Atabakhshian, V., Amir, S., and Loghman, A. "Nonlinear dynamical response of embedded fluid-conveyed micro-tube reinforced by BNNTs", Compos. Part B-Eng., 44(1), pp. 424-432 (2013).

17. Ghorbanpour Arani, A. and Hashemian, M. "Surface stress effects on dynamic stability of double-walled boron nitride nanotubes conveying viscose fluid based on nonlocal shell theory", Sci. Iran., 20(6), pp. 23562374 (2013).

18. Ghorbanpour Arani, A., Khoddami Maraghi, Z., and Haghparast, E. "The fluid structure interaction effect on the vibration and instability of a conveyed doublewalled boron nitride nanotube", Sci. Iran., 22(2), pp. 436-447 (2015).

19. Atabakhshian, V., Shoshtari, A.R., and Karimi, M. "Electro-thermal vibration of a smart coupled nanobeam system with an internal flow based on nonlocalel asticity theory", Physica B: Condensed Matter, 456, pp. 375-382 (2015).

20. Paidoussis, M.P. and Issid, N.T. "Dynamic stability of pipes conveying fluid", J. Sound. Vib., 33(3), pp. 267-294 (1974).

21. Panda, L.N. and Kar, R.C. "Nonlinear dynamics of a pipe conveying pulsating fluid with combination, principal parametric and internal resonances", Journal of Sound and Vibration, 309, pp. 375-406 (2008). 
22. Azrar, A., Azrar, L., and Aljinaidi, A.A. "Numerical modeling of dynamic and parametric instabilities of single-walled carbon nanotubes conveying pulsating and viscous fluid", Compos. Struct, 125(8), pp. 127143 (2015).

23. Liang, F. and Su, Y. "Stability analysis of a singlewalled carbon nanotube conveying pulsating and viscous fluid with nonlocal effect", Appl. Math. Model., 37, pp. 6821-6828 (2013).

24. Da, H.L., Wang, L., Qian, Q., and Ni, Q. "Vortexinduced vibrations of pipes conveying pulsating fluid", Ocean. Eng., 77, pp. 12-22 (2014).

25. Wang, L. "A further study on the non-linear dynamics of simply supported pipes conveying pulsating fluid", Int. J. Non. Linear Mech., 44, pp. 115-121 (2009).

26. Yang, K.S., Cheng, Y.C., Liu, M.C., and Shyu, J.C. "Micro pulsating heat pipes with alternate microchannel widths", Appl. Therm. Eng., 83, pp. 131-138 (2015).

27. Tubaldi, E., Amabili, V., and Païdoussis, M.P. "Fluidstructure interaction for nonlinear response of shells conveying pulsatile flow", J. Sound. Vib., 371, pp. 252-276 (2016).

28. Tubaldi, E., Amabili, M., and Paidoussis, M.P. "Nonlinear dynamics of shells conveying pulsatile flow with pulse-wave propagation: Theory and numerical results for a single harmonic pulsation", J. Sound Vib., 396, pp. 217-245 (2017).

29. Rafii-Tabar, H., Ghavanloo, E., and Fazelzadeh, S.A. "Nonlocal continuum-based modeling of mechanical characteristics of nanoscopic structures", Physics Reports, 638, pp. 1-97 (2016).

30. Mercan, K. and Civalek, O. "DSC method for buckling analysis of boron nitride nanotube (BNNT) surrounded by an elastic matrix", Compos. Struct., 143, pp. 300-309 (2016).

31. Akgoz, B. and Civalek, O. "Bending analysis of embedded carbon nanotubes resting on an elastic foundation using strain gradient theory", Acta Astronaut, 119, pp. 1-12 (2016).

32. Civalek, Ö. and Demir, Ç. "A simple mathematical model of microtubules surrounded by an elastic matrix by nonlocal finite element method", Appl. Math. Comput., 289, pp. 335-352 (2016).

33. Ghorbanpour Arani, A., Atabakhshian, V., Loghman, A., Shajari, A.R., and Amir, S. "Nonlinear vibration of embedded SWBNNTs based on nonlocal Timoshenko beam theory using DQ method", Physica B, 407, pp. $2549-2555$ (2012).

34. Alibeigi, B., Beni, Y.T., and Mehralian, F. "On the thermal buckling of magneto-electro-elastic piezoelectric nanobeams", Eur. Phys. J. Plus., 133, pp. 133138 (2018).
35. Institute of Electrical and Electronics Engineers, Standard on Piezoelectricity, Std, IEEE, New York (1978).

36. Ding, H.J. and Chen, W.Q. "Three dimensional problems of piezoelasticity", Nova Science, New York (2001).

37. Eringen, A.C. "Nonlocal polar elastic continua", INT. J. ENG. SCI., 10(1), pp. 1-16 (1972).

38. Eringen, A.C., Nonlocal Continuum Field Theories, Springer-Verlag, New York (2002).

39. Eringen, A.C. "On differential equations of nonlocal elasticity and solutions of screw dislocation and surface waves", J. Appl. Phys., 54, pp. 4703-4710 (1983).

40. Ke, L.L., Wang, Y.Sh., and Wang, Zh.D. "Nonlinear vibration of the piezoelectric nanobeams based on the nonlocal theory", Compos. Struct., 94(6), pp. 2038 2047 (2012).

41. Han, J.H. and Lee, I. "Analysis of composite plates with piezoelectric actuators for vibration control using layerwise displacement theory", Compos. Part B-Eng., 29(5), pp. 621-632 (1998).

42. Ke, L.L., Wang, Y.Sh., and Wang, Zh.D. "Nonlinear vibration of the piezoelectric nanobeams based on the nonlocal theory", Compos. Struct., 94(6), pp. 20382047 (2012).

43. Kurylov, Y. and Amabili, M. "Polynomial versus trigonometric expansions for nonlinear vibrations of circular cylindrical shells with different boundary conditions", J. Sound. Vib., 329(9), pp. 1435-1449 (2010).

44. Alinia, M.M. and Ghannadpour, S. "Nonlinear analysis of pressure loaded FGM plates", Compos. Struct., 88(3), pp. 354-359 (2009).

45. Yang, J., An Introduction to the Theory of Piezoelectricity, 9th Ed., Springer, Lincoln (2005).

46. Fox, R.W., Pritchard, P.J., and McDonald, A.T., Introduction to Fluid Mechanics, 4th Ed., Wiley, New York, USA (2008).

47. Paidoussis, M.P., Misra, A.K., and Chan, S.P. "Dynamics and stability of coaxial cylindrical shells conveying viscous fluid", J. Appl. Mech-T., ASME., 52(2), pp. 389-396 (1985).

48. Karniadakis, G., Beskok, A., and Aluru, N., Micro Flows Nano Flows: Fundamentals and Simulation, Springer-Verlag (2005).

49. Rashidi, V., Mirdamadi, H.R., and Shirani, E. "A novel model for vibrations of nanotubes conveying nanoflow", Comput. Mater. Sci., 51, pp. 347-352 (2012).

50. Shokouhmand, H., Isfahani, A.H.M., and Shirani, E. "Friction and heat transfer coefficient in micro and nano channels filled with potous media for wide range of Knudsen number", Int. Comm. Heat Mass, 37, pp. 890-894 (2010).

51. Irschik, H. and Holl, H. "The equations of Lagrange written for a non-material volume", Acta Mech, 153, pp. 231-248 (2002). 
52. Yang, J., Ke, L.L., and Kitipornchai, S. "Nonlinear free vibration of single-walled carbon nanotubes using nonlocal Timoshenko beam theory", Physica E, 42, pp. $1727-1735$ (2010).

53. Bolotin, V.V., The Dynamic Stability of Elastic Systems, Holden-Day, Inc, San Francisco, USA (1964).

54. Amabili, M. and Graziera, R. "Vibrations of circular cylindrical shells with nonuniform constraints, elastic bed and added mass. Part ii: Shells containing or immersed in axial flow", J. Fluid. Struct., 16(1), pp. 31-51 (2002).

55. Mohammadi, K., Rajabpour, A., and Ghadiri, M. "Calibration of nonlocal strain gradient shell model for vibration analysis of a CNT conveying viscous fluid using molecular dynamics simulation", Comp. Mater. Sci., 148, pp. 104-115 (2018).

\section{Biographies}

Vahid Atabakhshian received his BSc degree from the Islamic Azad University in Kashan, Kashan, Iran in 2009. He then received his MS degree from the University of Kashan, Kashan, Iran in 2013. He is currently a PhD Student at Bu-Ali Sina University in Hamedan, Hamedan, Iran. His current research interests are flow-induced vibration, smart materials, nanomechanics, nonlinear dynamic, buckling, and composites.

Alireza Shooshtari is an Associate Professor of Mechanical Engineering at Bu-Ali Sina University, Hamedan, Iran. He received his BS degree in Mechanical Engineering from Tehran University, Tehran, Iran and his MS and PhD degrees in Applied Mechanics from Bu-Ali Sina University, Hamedan, Iran and Tarbiat Modarres University, Tehran, Iran, respectively. He has been an academic staff in Mechanical Engineering Department, Bu-Ali Sina University since 2006. His research interests are in the field of dynamics and vibration, especially in nonlinear vibration and dynamics of smart structures, continuous systems, modal analysis, vibration of nanostructures, and composites structures. 